\section{Immune Suppression and Response to Ipilimumab: Assessing Risk-to-Benefit Ratio}

To THE EDITOR: In their recent article, Horvat et $\mathrm{al}^{1}$ evaluated the effects of immune-related adverse events (irAEs) in about 300 patients with metastatic melanoma treated with ipilimumab. In recent years, accumulating data suggested that ipilimumab supports the activation of the immune system, thus promoting antitumor immunity. ${ }^{2,3}$ However, immune system activation may induce in turn normal tissue injury (ie, irAEs), which occurs in about $60 \%$ to $80 \%$ of patients treated. ${ }^{1,2}$ The authors observed that both irAEs (any severity) and the administration of systemic immunosuppressive therapies (ie, corticosteroids) did not influence survival outcome. ${ }^{1}$ This information is useful in clinical practice for patients affected by metastatic melanoma treated with ipilimumab, in whom corticosteroid use is generally avoided. ${ }^{1}$

However, the following points of this paper deserve to be addressed. There is growing evidence showing that an (excessive) activation of the immune response, leading to immune-related toxicities, is associated with an improved response to immunotherapy. ${ }^{3,4}$ By this point of view, it would be interesting to assess the prognosis of patients who experienced severe irAEs with corticosteroid rescue. In addition, the authors should clarify if the patients with irAEs achieved a response to ipilimumab before the start of corticosteroid rescue. In the latter case we can hypothesize that, when an efficient immune reaction is activated by ipilimumab, administration of immunosuppressive therapies does not influence response to treatment. In fact, although the efficacy of systemic steroids in suppressing immune response is well known, the kinetics of development (time and dose relation) of such immunosuppressive effect are not completely clear. ${ }^{5,6}$ In this regard, knowledge of the schedule and the dose of corticosteroid that had been used would be useful information.
Improving knowledge of the relationship between systemic immune activation and clinical responses to immune therapies would be helpful from a clinical point of view. In fact, an effective immunotherapy should be continued even if patients experience irAEs, making rescue with corticosteroids not harmful for their activity. Further attempts are needed to improve the care of patients and their quality of life.

\section{Alice Indini, Massimo Di Nicola, Michele Del Vecchio, and Filippo De Braud}

Fondazione Istituto Di Ricovero e Cura a Carattere Scientifico Istituto Nazionale dei Tumori, Milan, Italy

\section{AUTHORS' DISCLOSURES OF POTENTIAL CONFLICTS OF INTEREST}

Disclosures provided by the authors are available with this article at www.jco.org.

\section{REFERENCES}

1. Horvat TZ, Adel NG, Dang TO, et al: Immune-related adverse events, need for systemic immunosuppression, and effects on survival and time to treatment failure in patients with melanoma treated with ipilimumab at Memorial Sloan Kettering Cancer Center. J Clin Oncol 33:3193-3198, 2015

2. Hodi FS, O'Day SJ, McDermott DF, et al: Improved survival with ipilimumab in patients with metastatic melanoma. N Engl J Med 363:711-723, 2010

3. Downey SG, Klapper JA, Smith FO, et al: Prognostic factors related to clinical response in patients with metastatic melanoma treated by CTL-associated antigen-4 blockade. Clin Cancer Res 13:6681-6688, 2007

4. Feng $Y$, Roy $A$, Masson $E$, et al: Exposure-response relationships of the efficacy and safety of ipilimumab in patients with advanced melanoma. Clin Cancer Res 19:3977-3986, 2013

5. Goel AP, Nguyen VH, Hamill-Ruth R: Use of a risk-stratification tool in identification of potential adrenal suppression preceding steroid injection therapy in chronic pain patients. Pain Med 10.1111/pme.12855 [epub ahead of print on August 4, 2015] doi:10.1111/pme.12855

6. Coutinho $\mathrm{AE}$, Chapman KE: The anti-inflammatory and immunosuppressive effects of glucocorticoids, recent developments and mechanistic insights. Mol Cell Endocrinol 335:2-13, 2011

DOI: 10.1200/JCO.2015.65.0028; published online ahead of print at www.jco.org on January 19, 2016 


\section{AUTHORS' DISCLOSURES OF POTENTIAL CONFLICTS OF INTEREST}

Immune Suppression and Response to Ipilimumab: Assessing Risk-to-Benefit Ratio

The following represents disclosure information provided by authors of this manuscript. All relationships are considered compensated. Relationships are self-held unless noted. I = Immediate Family Member, Inst = My Institution. Relationships may not relate to the subject matter of this manuscript. For more information about ASCO's conflict of interest policy, please refer to www.asco.org/rwc or jco.ascopubs.org/site/ifc.

Alice Indini

No relationship to disclose

Massimo Di Nicola

No relationship to disclose

Michele Del Vecchio

Honoraria: Roche, Novartis, Bristol-Myers Squibb, MSD

Consulting or Advisory Role: Roche, Novartis, MSD

Research Funding: Roche (Inst), MSD (Inst), Novartis (Inst), Bristol-

Myers Squibb (Inst)

\section{Filippo De Braud}

Consulting or Advisory Role: Bristol-Myers Squibb, Novartis, Roche, Merck

Research Funding: Bristol-Myers Squibb (Inst), Novartis (Inst), Roche (Inst), Merck (Inst) 